\title{
Avaliação genética do crescimento de bovinos Nelore Mocho, por meio de modelos de multicaracterísticas ${ }^{1}$
}

\author{
Isabella Coutinho de Barros ${ }^{2}$, Rodrigo Reis Mota ${ }^{3 *}$, Luciano Pinheiro da Silva ${ }^{4}$, Paulo Luiz Souza Carneiro ${ }^{2}$, \\ Raimundo Martins Filho ${ }^{5}$, Carlos Henrique Mendes Malhado ${ }^{2}$
}

\author{
$10.1590 / 0034-737 X 201865050004$
}

\section{RESUMO}

Objetivou-se, com este trabalho, estimar parâmetros genéticos para características do crescimento de animais da raça Nelore Mocho. Foram utilizados pesos corporais (Kg) ajustados aos 205 (P205), 365 (P365) e 550 (P550) dias. Adotou-se modelo multicaraterísticas ajustado para o efeito fixo dos grupos de contemporâneos, idade da vaca ao parto (covariável) e, para os efeitos aleatórios genético direto, genético materno, ambiente permanente materno e residual. As variâncias genéticas diretas $\left(98,80,276,38\right.$ e 547,52 $\left.\mathrm{Kg}^{2}\right)$ e maternas $\left(61,77,64,63\right.$ e 79,85 $\left.\mathrm{Kg}^{2}\right)$, elevaram-se com o avanço das idades. Observou-se redução das variâncias de ambiente permanente materno de P205 para P365 e aumento para P550. As herdabilidades diretas, e seus erros padrão, para P205, P365 e P550 foram respectivamente de 0,21 $\pm 0,01 ; 0,38 \pm 0,00$ e $0,39 \pm 0,00$. As herdabilidades maternas foram maiores no início da vida dos animais $(0,13 \pm 0,01,0,08$ $\pm 0,00$ e $0,05 \pm 0,00)$. As correlações genéticas diretas foram superiores a 0,94 . As correlações fenotípicas apresentaram valores de moderados a altos $(0,50$ a 0,87$)$. As correlações genéticas maternas e de ambiente permanente materno foram superiores a 0,91. Ganhos genéticos são esperados, ao se usarem as características do crescimento como critérios de seleção. A existência de variabilidade genética permite a seleção em idades menores, possibilitando a redução do intervalo de gerações e aumento do ganho genético.

Palavras-chave: componentes de variância; correlação genética; ganho genético; herdabilidade.

\section{ABSTRACT}

\section{Genetic evaluation of the growth of Polled Nellore via multi-trait models}

We aimed to estimate genetic parameters for growth traits of Polled Nellore beef cattle. Adjusted weights at 205 (W205), 365 (W365), and 450 (W450) days were used. The effects of contemporary groups (fixed), dam age at calving (covariate), and additive genetic, maternal genetic, maternal permanent environmental, and residual (random) were fit under a multi-trait model. The additive genetic $\left(98.80,276.38\right.$, and $\left.547.52 \mathrm{Kg}^{2}\right)$ and maternal genetic $(61.77,64.63$, and $79.85 \mathrm{Kg}^{2}$ ) variance estimates increased as the age of the animals increased. A decrease was observed from W205 to W365 for the maternal permanent environmental effect. Direct heritability estimates were $0.21 \pm 0.01 ; 0.38 \pm 0.00$, and $0.39 \pm 0.00$ for W205, W365, and W450, respectively. The maternal heritabilities were higher at lower ages $(0.13 \pm 0.01$, $0.08 \pm 0.00$, and $0.05 \pm 0.00$ ). Genetic correlations were higher than 0.94 , whereas phenotypic correlations were moderate to high, between 0.50 and 0.87 . The maternal genetic and maternal permanent environmental correlations were higher than 0.91. Potential genetic gains might be expected when using these growth traits as selection criteria. The verified genetic variability may allow the selection of animals at lower ages (e.g., weaning), enabling the reduction of generation interval and increase of genetic gain.

Keywords: genetic correlation; genetic gain; heritability; variance components.

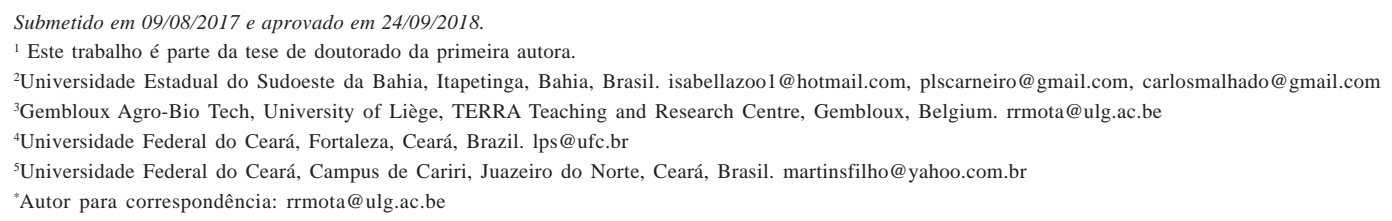




\section{INTRODUÇÃO}

A pecuária de corte brasileira busca alternativas para conseguir atender a crescente demanda de carne no mercado mundial. Segundo estimativas da FAO (2017), o mundo precisará aumentar a produção de carne bovina, em $34 \%$, até 2030. Para alcançar esse objetivo, é necessário o aumento do grau de eficiência econômico-produtiva e uma das ferramentas que pode ser utilizada é o melhoramento genético.

A avaliação genética de bovinos de corte depende, essencialmente, da disponibilidade das estimativas de parâmetros genéticos para as características de interesse econômico. Segundo Albuquerque \& Faro (2008), componentes de variância são estimados, considerando-se os pesos às idades padrão, como ao nascimento, à desmama e ao ano.

Nesse sentido, as avaliações genéticas em gado de corte vêm utilizando registros de pesos corporais (Boligon et al., 2009). Estes registros são importantes para a avaliação da eficiência dos sistemas de produção, além de constantemente serem considerados como critério de seleção em programas de melhoramento genético (Baldi et al., 2010).

Os modelos bicaracterísticas são amplamente utilizados em análises conjuntas de pesos ajustados às idades padrão (Garnero et al., 2010; Pedrosa et al., 2010). Esses modelos adotam matrizes de covariâncias sem restrições, consideram cada medida do animal como uma característica distinta e fornecem informações sobre as relações lineares existentes entre as idades (Boligon et al., 2009).

A maior parte da carne bovina brasileira é oriunda de sistemas de produção extensivos, em regime de pastagens e com rebanhos constituídos de raças zebuínas, adaptadas às condições de criação em climas tropicais. Segundo a Associação Brasileira dos Criadores de Zebu (ABCZ, 2017), das raças zebuínas, a Nelore corresponde a quase $80 \%$ do rebanho, no Brasil. Deve-se ainda destacar que o Nelore Mocho encontra-se na segunda posição em número de registros pela $\mathrm{ABCZ}$ e, atualmente, representa $13,4 \%$ dos animais zebuínos com registro genealógico definitivo.

No Brasil, o Nelore Mocho é normalmente criado separadamente do Nelore comum, e apresenta vantagens, como menores riscos para os trabalhadores durante a lida e transporte. Pesos ajustados às idades padrão permitem avaliações genéticas do Nelore, no Brasil (Lopes et al., 2011; Santos et al., 2005); porém, dados e estudos em populações de Nelore Mocho, nas diversas regiões do Brasil (Amaral et al., 2014; Garcia et al., 2003; Lopes et al., 2013), ainda são insuficientes para estabelecimento de programas de melhoramento da raça. Por essa razão, objetivou-se, neste trabalho, estimar parâmetros genéticos para desenvolvimento ponderal para bovinos Nelore Mocho, criados na região nordeste do Brasil.

\section{MATERIAL E MÉTODOS}

Foram utilizados, neste estudo, registros de pesos e genealogia de bovinos Nelore Mocho, nascidos entre os anos de 1975 e 2011, na região nordeste do Brasil. Os dados são provenientes da ABCZ. Os grupos de contemporâneos (GC) foram definidos por animais do mesmo sexo, nascidos na mesma fazenda, na mesma época e ano e sob mesmo regime alimentar. GC com menos de três animais foram excluídos das análises. $\mathrm{O}$ arquivo de dados foi composto por pesos corporais $(\mathrm{Kg})$ ajustados às idades de 205 (P205), 365 (P365) e 550 (P550) dias de idade (Tab. 1). Para o ajuste de P205, P365 e P550, foram consideradas as pesagens realizadas entre 60 e 205, 206 e 365, 366 e 653 dias, respectivamente.

A estimação dos componentes de covariância e dos parâmetros genéticos foram realizados pelo Método da Máxima Verossimilhança Restrita (REML), com o programa airemlf90 (http://nce.ads.uga.edu/), utilizando-se os algoritmos EM e AI-Modificado. Foi utilizado, como critério de convergência, o padrão do programa $\left(10^{-5}\right)$. A covariância entre os efeitos genético direto e genético materno foi assumida como zero.

Adotou-se modelo animal ajustado com três caraterísticas de desenvolvimento ponderal (P205, P365 e P550), que continha o efeito fixo de grupos contemporâneos, a covariável idade da vaca ao parto e os efeitos aleatórios, genético direto, genético materno, ambiente permanente materno e residual.

O modelo pode ser descrito, matricialmente, da seguinte forma:

$\mathrm{y}=\mathrm{X} \beta+\mathrm{Z}_{1} \mathrm{~d}+\mathrm{Z}_{2} \mathrm{~m}+\mathrm{Z}_{3} \mathrm{mp}+\mathrm{e}$,

em que y = matriz de observações para P205, P365 e P550; $\beta=$ matriz de efeitos fixos; $d=$ matriz de efeitos aleatórios genéticos diretos; $\mathrm{m}=$ matriz de efeitos aleatórios genéticos maternos; $\mathrm{mp}=$ matriz de efeitos aleatórios de ambiente permanente materno; $X=$ matriz de incidência dos efeitos fixos; $Z_{1}, Z_{2}$ e $Z_{3}=$ matrizes de incidência, respectivamente, aos efeitos aleatórios genético direto, genético materno e de ambiente permanente; $\mathrm{e}=$ matriz de efeitos aleatórios residuais.

As seguintes pressuposições foram assumidas:

$$
\begin{aligned}
& {\left[\begin{array}{c}
\mathrm{y} \\
\mathrm{d} \\
\mathrm{m} \\
\mathrm{mp} \\
\mathrm{e}
\end{array}\right]=\left[\begin{array}{c}
\mathrm{X} \beta \\
0 \\
0 \\
0 \\
0
\end{array}\right]} \\
& \mathrm{e}, \mathrm{V}\left[\begin{array}{c}
\mathrm{d} \\
\mathrm{m} \\
\mathrm{mp} \\
\mathrm{e}
\end{array}\right]=\left[\begin{array}{cccc}
\mathrm{G}_{\mathrm{d}} \otimes \mathrm{A} & 0 & 0 & 0 \\
0 & \mathrm{G}_{\mathrm{m}} \otimes \mathrm{A} & 0 & 0 \\
0 & 0 & \mathrm{G}_{\mathrm{mp}} \otimes \mathrm{I}_{\mathrm{v}} & 0 \\
0 & 0 & 0 & \mathrm{R} \otimes \mathrm{I}_{\mathrm{n}}
\end{array}\right]
\end{aligned}
$$


em que $\mathrm{G}_{\mathrm{d}}=$ matriz de covariâncias genéticas diretas; $\mathrm{G}_{\mathrm{m}}=$ matriz de covariâncias genéticas maternas; $\mathrm{G}_{\mathrm{mp}}=$ matriz de covariâncias de ambiente permanente materno; $\mathrm{R}=$ matriz de covariâncias residuais; $\mathrm{A}=$ matriz dos numeradores dos coeficientes de parentesco de Wright; $\mathrm{I}_{\mathrm{v}}=$ matriz identidade, cuja ordem (v) é igual ao número de mães; $\mathrm{I}_{\mathrm{n}}=$ matriz identidade, cuja ordem (n) é igual ao número de observações; $\otimes=$ operador do produto de Kronecker.

\section{RESULTADOS E DISCUSSÃO}

As estimativas de variância genética direta $\left(\sigma^{2}{ }_{\mathrm{d}}\right)$ foram crescentes com as idades. Os valores dessas estimativas foram de 98,80, 276,38 e 547,52 $\mathrm{Kg}^{2}$, para P205, P365 e P550, respectivamente (Tab. 2). Essa mesma tendência de crescimento das estimativas de variância com a idade foi anteriormente descrita na literatura para animais da raça Nelore (Boligon et al., 2009; Pedrosa et al., 2014). Normalmente, valores elevados de variância genética direta denotam significativa variabilidade e que a população responderá à seleção para essas características. No entanto, se a variância ambiental for maior que a genética, mais difícil torna-se a obtenção de ganho genético.

As estimativas de variância genética materna $\left(\sigma_{\mathrm{m}}^{2}\right)$ também foram crescentes, P205 (61,77 Kg $\left.{ }^{2}\right)$, P365 $\left(64,63 \mathrm{Kg}^{2}\right)$ e P550 (79,85 $\left.\mathrm{Kg}^{2}\right)$. O crescimento da variância genética materna é esperada pelo menos até o desmame, uma vez que esse período corresponde ao de maior influência da mãe. Comportamento semelhante foi observado por Valente et al. (2008), em bovinos Nelore comum.

As estimativas de variância de ambiente permanente materno $\left(\sigma_{\mathrm{mp}}^{2}\right)$ apresentaram redução à desmama (P205), ao ano (P365), seguido de posterior aumento para P550 (Tab. 2). Isso pode ser justificado pelo número médio de crias por vaca $(\bar{X}=1.54)$. A literatura tem constantemente demonstrado, para diferentes raças de bovinos de corte, que o efeito de ambiente permanente materno é de difícil estimação, quando o número médio de crias por vaca é inferior a dois (Souza et al., 2011; Menezes et al., 2013). Schaeffer (2004) observou que variâncias de ambiente permamente materno tendem a zero, quando com o número médio de crias por vaca é igual a, ou menor que, dois (02). Dados com estrutura limitada, caso deste estudo, impedem a estimabilidade biológica de alguns parâmetros, e fazem necessárias várias gerações de dados com máxima relação de parentesco entre animais e suas respectivas mães. (Maniatis \& Pollott, 2003).

Houve crescimento constante nas estimativas de variância fenotípica $\left(\sigma_{p}^{2}\right.$; Tab. 2). Esses resultados corroboram os obtidos por Sousa Júnior et al. (2010), com animais Tabapuã. O aumento foi atribuído pelos autores à progressão das variâncias genéticas diretas e residuais, ao longo do crescimento.

As estimativas de herdabilidade direta $\left(\mathrm{h}^{2}{ }_{\mathrm{d}}\right)$ foram de 0,21 $\pm 0,01,0,38 \pm 0,00$ e 0,39 $\pm 0,00$ para P205, P365 e P550, respectivamente (Tab. 2). A tendência de crescimento das estimativas de também foi relatada por Caires et al. (2012). Os autores avaliaram animais Tabapuã e observaram valor superior para 550 dias de idade $(0,36)$. Valores semelhantes de $\mathrm{h}^{2}{ }_{\mathrm{d}}$ foram reportados por Malhado et al. (2005), para P205 (0,24), e por Araújo et al. (2014), nas três idades, de $0,36,0,31$ e 0,38, respectivamente, para os 210, 365 e 550 dias. Lira et al. (2008), em 67 trabalhos com zebuínos, encontraram estimativas médias de herdabilidade direta de 0,28, 0,31 e 0,35, para P205, P365 e P550, respectivamente. Teoricamente, respostas moderadas e semelhantes para a seleção são esperadas, ao se selecionar para

Tabela 1: Descrição dos dados para pesos pré-ajustados aos 205 (P205), 365 (P365) e 550 (P550) dias de idade

\begin{tabular}{lcccccccc}
\hline Pesos & N & Touros & Vacas & GC & Média $(\mathbf{K g})$ & DP $(\mathbf{K g})$ & Mínimo $(\mathbf{K g})$ & Máximo $(\mathbf{K g})$ \\
\hline P205 & 8.418 & 47 & 73 & 635 & 173,35 & 28,82 & 25 & 310 \\
P365 & 8.418 & 47 & 73 & 635 & 234,38 & 41,19 & 72 & 499 \\
P550 & 8.418 & 47 & 73 & 635 & 306,24 & 66,45 & 88 & 734 \\
\hline
\end{tabular}

GC: grupo de contemporâneos; N: número de observações; DP: desvio padrão.

Tabela 2: Estimativas de variâncias genética direta $\left(\sigma_{\mathrm{d}}^{2}\right)$, genética materna $\left(\sigma_{\mathrm{m}}^{2}\right)$, de ambiente permanente materno $\left(\sigma_{\mathrm{mp}}^{2}\right), \operatorname{residual}\left(\sigma_{\mathrm{e}}^{2}\right)$ e fenotípica $\left(\sigma_{\mathrm{p}}^{2}\right)$, herdabilidade direta $\left(\mathrm{h}_{\mathrm{d}}^{2}\right)$, herdabilidade materna $\left(\mathrm{h}_{\mathrm{m}}^{2}\right)$, proporção da variância de ambiente permanente materno $\left(\mathrm{mp}^{2}\right)$ e residual $\left(\mathrm{e}^{2}\right)$ sobre a variância fenotípica

\begin{tabular}{lccccc}
\hline Pesos & $\boldsymbol{\sigma}_{\mathbf{d}}$ & $\boldsymbol{\sigma}_{\mathbf{m}}$ & $\boldsymbol{\sigma}_{\mathbf{m p}}$ & $\boldsymbol{\sigma}_{\mathbf{e}}$ & $\boldsymbol{\sigma}_{\mathbf{p}}$ \\
\hline P205 & $98,80 \pm 3,95$ & $61,77 \pm 3,56$ & $44,05 \pm 2,66$ & $250,54 \pm 6,84$ & $455,16 \pm 6,61$ \\
P365 & $276,38 \pm 10,44$ & $64,63 \pm 3,75$ & $40,95 \pm 2,48$ & $327,72 \pm 9,08$ & $709,67 \pm 7,23$ \\
P550 & $547,52 \pm 20,62$ & $79,85 \pm 4,70$ & $45,78 \pm 2,80$ & $740,74 \pm 18,73$ & $1413,90 \pm 9,22$ \\
\hline Pesos & $\mathbf{h}^{2}{ }_{\mathbf{d}}$ & $\mathbf{h}^{2}{ }_{\mathbf{m}}$ & $\mathbf{m p}^{2}$ & $\mathbf{e}^{2}$ & \\
-P205 & $0,21 \pm 0,01$ & $0,13 \pm 0,01$ & $0,10 \pm 0,01$ & $0,14 \pm 0,01$ & - \\
P365 & $0,38 \pm 0,00$ & $0,08 \pm 0,00$ & $0,06 \pm 0,00$ & $0,33 \pm 0,01$ & - \\
P550 & $0,39 \pm 0,00$ & $0,05 \pm 0,00$ & $0,03 \pm 0,00$ & $0,67 \pm 0,00$ & - \\
\hline
\end{tabular}

Rev. Ceres, Viçosa, v. 65, n.5, p. 402-406, set/out, 2018 
pesos, nas três idades avaliadas, por causa da similaridade das herdabilidades. No entanto, vale ressaltar que, mesmo se as herdabilidades forem idênticas, o ganho genético pode ser diferente, por conta de diferentes intensidades de seleção e das diferenças na variância fenotípica.

Estimativas similares de herdabilidades maternas $\left(\mathrm{h}^{2}{ }_{\mathrm{m}}\right)$, ao desmame, em populações de Nelore Mocho (P205; Tab. 2) foram anteriormente reportadas na literatura (Amaral et al., 2014; Malhado et al., 2008). Os autores supracitados

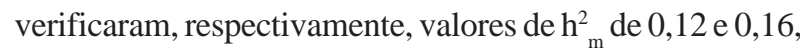
no Estado da Bahia. Esses resultados destacam a maior importância desse efeito na fase de crescimento dos animais e para a população em estudo. O efeito materno pode proporcionar um ambiente ideal a suas proles para seu desenvolvimento e, às mães, uma variação na sua produção de leite, capacidade de defesa ou de busca por alimentos. A tendência de descréscimo da herdabilidade materna com o aumento da idade foi amplamente reportada na literatura (Boligon et al., 2011; Caetano et al., 2013).

As estimativas de proporção da variância de ambiente permanente materno $\left(\mathrm{mp}^{2}\right)$, em relação à variância fenotípica total, apresentaram valor máximo à desmama (Tab. 2). Isto ocorreu por causa da diminuição da variância de ambiente materno após a desmama (205 - 550 dias) e é esperado, pelo fato de o animal, teoricamente, não sofrer mais influência materna. O mesmo comportamento foi relatado por Mota $e t$ al. (2013), avaliando animais da raça Simental, os quais relataram valores de $\mathrm{mp}^{2} \mathrm{de} 0,13$, ao desmame, de 0,07 , aos 12 meses e de 0,03 , aos 18 meses de idade.

As estimativas de proporção de variância residual $\left(\mathrm{e}^{2}\right)$ aumentaram com o aumento das idades (Tab. 2). Esses valores foram de 0,14, 0,33 e 0,67, para P205, P365 e P550, respectivamente. Essa tendência também foi relatada por Garnero et al. (2010), avaliando animais Nelore por meio de modelos uni e bicaracterísticos.

As estimativas de correlações genéticas diretas foram altas e positivas, indicando alto grau de associação entre as caraterísticas avaliadas (Tab. 3). Altas correlações indicam que os grupos de genes responsáveis por maiores pesos aos 205 dias de idade são praticamente os mesmos nas demais idades. Além disso, a seleção pode ser feita mais cedo, durante o ciclo de vida dos animais, i.e., a seleção para o aumento do peso em idade mais jovem leva ao aumento do peso adulto. Valores de correlações gené-

Tabela 3: Estimativas de correlações genéticas aditivas diretas (acima da diagonal) e fenotípicas (abaixo da diagonal), obtidas para pesos aos 205 (P205), 365 (P365), 550 (P550) dias de idade

\begin{tabular}{lccc}
\hline Pesos & P205 & P365 & P550 \\
\hline P205 & - & $0,97 \pm 0,04$ & $0,94 \pm 0,04$ \\
P365 & $0,73 \pm 0,00$ & - & $0,99 \pm 0,04$ \\
P550 & $0,50 \pm 0,00$ & $0,87 \pm 0,00$ & - \\
\hline
\end{tabular}

Tabela 4: Estimativas de correlações genéticas maternas (acima da diagonal) e ambiente permanente materno (abaixo da diagonal), obtidas para pesos aos 205 (P205), 365 (P365), 550 (P550) dias de idade

\begin{tabular}{lccc}
\hline Pesos & P205 & P365 & P550 \\
\hline P205 & - & $0,97 \pm 0,02$ & $0,92 \pm 0,02$ \\
P365 & $0,97 \pm 0,01$ & - & $0,98 \pm 0,03$ \\
P550 & $0,92 \pm 0,01$ & $0,98 \pm 0,02$ & - \\
\hline
\end{tabular}

ticas diretas, positivas e de grande magnitude, foram anteriormente reportadas em uma população de Nelore Mocho, no Brasil (Santos et al., 2005).

As estimativas de correlações fenotípicas para as idades avaliadas foram de média a alta magnitudes $(0,50-$ $0,87)$ e maiores em idades mais próximas. Maiores correlações em idades mais próximas é uma tendência amplamente reportada na literatura, não apenas em Nelore mocho (Santos et al., 2005), mas também em outras raças, como na Simmental (Mota et al., 2013) e até mesmo em outras espécies, como caprinos (Sarmento et al., 2003).

As correlações maternas apresentaram valores superiores a 0,91 (Tab. 4). Isso também sugere que os genes relacionados com o efeito materno, ao longo da trajetória de crescimento, são praticamente os mesmos. Logo, a seleção baseada em uma das idades irá afetar a todas, de maneira similar. Albuquerque \& Meyer (2001) e Baldi et al. (2010) encontraram estimativas de alta magnitude e positivas para as mesmas caraterísticas. As estimativas de correlações de ambiente permanente materno também foram altas (> 0,90; Tab. 4). Esses resultados corroboram os de Mota et al. (2013) e Menezes et al. (2013), que relataram estimativas superiores a 0,90 entre a desmama e os dois anos de idade. Por fim, as correlações de ambiente permanente materno, entre todos os pares de pesos, situaramse acima de 0,90, o que indica alto grau de associação entre as três características.

Constatou-se, nesta pesquisa, que o uso de qualquer uma das características analisadas (P205, P365 ou P450) como critério de seleção resultará em resposta correlacionada. Porém, o mais indicado seria fazer seleção na menor idade possível, para que se possa aumentar o ganho genético e ao mesmo tempo diminuir o intervalo entre gerações.

\section{CONCLUSÕES}

Ganhos genéticos podem ser alcançados ao se utilizar as características do crescimento como critério de seleção, em programas de melhoramento da variedade mocha da raça Nelore.

A existência de variabilidade genética para a seleção por meio do efeito genético direto e a seleção em idades menores possibilita a redução do intervalo de geração e consequente aumento no ganho genético. 


\section{FINANCIAMENTO EAPOIO}

Os autores agradecem à FAPESB, pelo suporte financeiro, e à Associação Brasileira de Criadores de Zebu (ABCZ), pela concessão dos dados.

\section{REFERÊNCIAS}

ABCZ - Associação Brasileira dos Criadores de Zebu (2017) Disponível em <http://www.abcz.org.br/>. Acessado em: 02 de janeiro de 2017.

Albuquerque L \& Meyer K (2001) Estimates of covariance functions for growth from birth to 630 days of age in Nelore cattle. Journal of Animal science, 79:2776-2789.

Albuquerque LG \& Faro LE (2008) Comparações entre os valores genéticos para características de crescimento de bovinos da raça Nelore preditos com modelos de dimensão finita ou infinita. Revista Brasileira de Zootecnia, 37:238-246.

Amaral RS, Carneiro PLS, Ambrosini DP \& Malhado CHM (2014) Tendências parâmetros fenotípicos e genéticos de características de crescimento em bovinos Nelore mocho do Nordeste brasileiro. Revista Brasileira de Saúde e Produção Animal, 15:261271 .

Araújo CV, Lôbo RB, Figueiredo LGG, Mousquer CJ, Laureano MMM, Bittencourt TCBS \& Araújo SI (2014) Estimates of genetic parameters of growth traits of Nellore cattle in the Midwest region of Brazil. Revista Brasileira de Saúde e Produção Animal, 15:846-853.

Baldi F, Albuquerque L \& Alencar M (2010) Random regression models on Legendre polynomials to estimate genetic parameters for weights from birth to adult age in Canchim cattle. Journal of Animal Breeding and Genetics, 127:289-299.

Boligon A, Baldi F \& Albuquerque LG (2011) Estimates of genetic parameters for scrotal circumference using random regression models in Nelore cattle. Livestock Science, 137:205-209.

Boligon A, Mercadante M, Baldi F, Lôbo R \& Albuquerque L (2009) Multi-trait and random regression mature weight heritability and breeding value estimates in Nelore cattle. South African Journal of Animal Science, 39:145-148.

Caetano S, Savegnago R, Boligon A, Ramos S, Chud T, Lôbo R \& Munari D (2013) Estimates of genetic parameters for carcass growth and reproductive traits in Nellore cattle. Livestock Science, 155:01-07.

Caires DN, Malhado CHM, Souza LA, Neto T, Rezende M, Carneiro PLS \& Martins Filho R (2012) Tabapuã breed in Northeastern Brazil: genetic progress and population structure. Revista Brasileira de Zootecnia, 41:1858-1865.

FAO - Organização das Nações Unidas para a Alimentação e Agricultura (2017) Disponível em: 〈http://www.fao.org>. Acessado em: 26 de janeiro de 2017.

Garcia FQ, Ferraz Filho P, Souza J \& Silva L (2003) Tendência dos efeitos genéticos diretos e maternos do peso a desmama de bovinos da raça Nelore Mocha na região pecuária Campo Grande e Dourados Mato Grosso do Sul. Archives of Veterinary Science, 8:93-97.

Garnero A, Muñoz M, Marcondes C, Lôbo RB, Lira T \& Gunski R (2010) Estimação de parâmetros genéticos entre pesos pré e pós-desmama na raça Nelore. Archivos de Zootecnia, 59:307310 .

Lira T, Rosa E \& Garnero A (2008) Parâmetros genéticos de características produtivas e reprodutivas em zebuínos de corte (revisão). Ciência Animal Brasileira, 9:01-22.
Lopes FB, Magnabosco CU, Paulini F, Silva MC, Miyagi ES \& Lôbo RB (2013) Genetic analysis of growth traits in polled Nellore cattle raised on pasture in tropical region using Bayesian approaches. PloS one, 8:1-6.

Lopes FB, Santos GCJ, Marques EG, Silva MC \& Ferreira JL (2011) Tendência genética para características relacionadas a velocidade de crescimento em bovinos Nelore da região Norte do Brasil. Revista Ciência Agronômica, 43:362-367.

Malhado CHM, Carneiro PLS, Martins Filho R, Azevêdo DMMR, Facó O, Machado CHC \& Piccinin A (2005) Tendência e parâmetros genéticos para o peso aos 205 dias de idade em bovinos da raça Nelore Mocho no estado da Bahia. Revista Científica de Produção Animal, 7:28-34.

Malhado CHM, Carneiro PLS, Pereira DG, Martins Filho R \& Souza J (2008) Progresso genético e estrutura populacional do rebanho Nelore no Estado da Bahia. Pesquisa Agropecuária Brasileira, 43:1163-1169.

Maniatis N \& Pollott GE (2003) The impact of data structure on genetic (co)variance components of early growth in sheep, estimated using an animal model with maternal effects. Journal of Animal Science, 81:101-108.

Menezes GRO, Torres RA, Torres Júnior RAA, Silva LOC, Gondo A \& Euclydes RF (2013) Estimation of genetic parameters for growth traits in Tabapuã cattle using a multi-trait model. Revista Brasileira de Zootecnia, 42:570-574.

Mota RR, Marques LFA, Lopes PS, Silva LP, Araújo Neto FR, Resende MDV \& Torres RA (2013) Genetic evaluation using multi-trait and random regression models in Simmental beef cattle. Genetics and Molecular Research, 12:2465-2480.

Pedrosa VB, Eler JP, Ferraz JBS \& Pinto LFB (2014) Utilização de modelos unicaracterística e multicaracterística na estimação de parâmetros genéticos na raça Nelore. Arquivo Brasileiro Medicina Veterinária e Zootecnia, 66:1802-1812.

Pedrosa VB, Eler JP, Ferraz JBS, Silva JAI, Ribeiro S, Silva MR \& Pinto LFB (2010) Parâmetros genéticos do peso adulto e características de desenvolvimento ponderal na raça Nelore. Revista Brasileira de Saúde e Produção Animal, 11:104-113.

Santos P, Malhado C, Carneiro P, Martins Filho R, Azevedo D, Cunha E \& Ferraz Filho P (2005) Correlação genética fenotípica e ambiental em características de crescimento de bovinos da raça Nelore variedade Mocha. Archives of Veterinary Science, 10:55-60.

Sarmento JLR, Pimenta Filho EC, Ribeiro MN \& Martins Filho R (2003) Efeitos ambientais e genéticos sobre o ganho em peso diário de bovinos Nelore no estado da Paraíba. Revista Brasileira de Zootecnia. 32:325-330.

Schaeffer (2004) Application of random regression models in animal breeding. Livestock Production Science, 86:35-45.

Sousa Júnior SC, Oliveira SMP, Albuquerque LG, Boligon AA \& Martins Filho R (2010) Estimação de funções de covariância para características de crescimento da raça Tabapuã utilizando modelos de regressão aleatória. Revista Brasileira de Zootecnia, 39:1037-1045.

Souza J, Silva L, Gondo A, Freitas J, Malhado CM, Sereno J \& Lamberson W (2011) Parâmetros e tendência genética de peso de bovinos criados a pasto no Brasil. Archivos de Zootecnia, 60:457-465.

Valente B, Silva M, Silva L, Bergmann J, Pereira J, Fridrich A \& Corrêa G (2008) Estruturas de covariância de peso em função da idade de animais Nelore das regiões Sudeste e Centro-Oeste do Brasil. Arquivo Brasileiro Medicina Veterinária e Zootecnia, $60: 389-400$ 